\title{
Management of pain with regional analgesia
}

\author{
JOHN J. BONICA \\ M.D., D.Sc., F.F.A.R.C.S. \\ Department of Anesthesiology, University of Washington, Seattle, Washington 98195, U.S.A.
}

\section{Introduction}

Regional analgesia has been used for the management of acute pain for nearly a century. Local analgesia and somatic nerve blocks interrupt nociceptive input at its source or block nociceptive fibres in peripheral nerves. Blockade also interrupts the afferent limb of abnormal reflex mechanisms that may contribute to the pathogenesis of some pain syndromes. Moreover, since sympathetic fibres destined for somatic structures, particularly the limbs, course through somatic spinal nerves, blocking these may eliminate the sympathetic hyperactivity that often contributes to the pathogenesis of certain pain syndromes. Local anaesthetics in low concentration block the unmyelinated C and B fibres and small myelinated A delta fibres without blocking somatic motor function. On the other hand, in certain conditions it may be useful to block somatomotor nerves to relieve severe muscle spasm. By producing one or more of these effects, there is often prompt pain relief lasting for varying lengths of time depending on the concentration and characteristics of the local anaesthetic used. In certain conditions, pain relief outlasts by hours and sometimes days and weeks the transient pharmacological action of local anaesthetics. It has been suggested that block of sensory input for several hours stops the selfsustaining activity of the neurone pools in the neuraxis that may be responsible for some chronic pain states (Bonica, 1953; Melzack and Wall, 1980).

\section{Indications for clinical application}

Regional analgesic techniques can be used as diagnostic, prognostic, prophylactic and therapeutic tools (Bonica, 1958, 1974).

\section{Diagnostic blocks}

Certain nerve blocks are useful to help ascertain specific nociceptive pathways, to differentiate referred from local pain, and help determine the possible mechanisms of chronic pain states. It is also useful in the differential diagnosis of the site and cause of the pain, and in determining the patient's reaction if the pain is eliminated (Bonica, 1953, 1958, $1959,1974)$. Block of the appropriate nerves helps differentiate trigeminal neuralgia from atypical facial neuralgia, neuralgia involving the third division of trigeminal nerve from glossopharyngeal or vagal neuralgia, and pain caused by visceral disease from pain of somatic origin. For example, complete relief of chest or epigastric pain following intercostal nerve block at the midaxillary line suggests the pain is of somatic origin in the chest or abdominal wall, whereas lack of relief suggests it is a pain referred from viscera (Bonica, 1953).

\section{Prognostic blocks}

Properly applied, certain nerve blocks are used to predict the effects of prolonged interruption by injection of neurolytic agents or by neurosurgical section (Bonica, 1953, 1959, 1974). Moreover, prognostic blocks give the patient an opportunity to experience the numbness and other side effects that follow surgery or neurolytic block, and help the patient decide whether or not to have the procedure. Although clinical evidence suggests this tool has certain limitations in predicting the long term effects of spinal rhizotomy, it is still useful especially when prolonged interruption is done in patients with cancer.

\section{Prophylactic blocks}

A variety of nerve blocks are used to prevent pain and the delay of normal functional activity that follows trauma, infections or operations. In some centres, nerve block procedures are considered one of the most efficient methods to control postoperative or post-traumatic pain. This facilitates earlier functional rehabilitation and helps to prevent complications. Moreover, there is evidence that analgesia achieved with regional block for several days decreases the incidence of reflex sympathetic dystrophy and other chronic pain syndromes (Bonica, 1953, 1959; Drucken et al., 1959). 


\section{Therapeutic blocks}

Local anaesthesia and nerve blocks using local anaesthetics are effective in treating self-limiting disease accompanied by severe pain and in breaking up the so-called vicious circle in patients with causalgia and other reflex sympathetic dystrophy, myofascial syndromes and reflex muscle spasm. It provides symptomatic relief to permit other therapeutic measures or to use as an adjunct to other therapeutic modalities. Therapeutic blocks with neurolytic agents are usually limited to patients with cancer pain, although they may be indicated in selected patients with trigeminal neuralgia, causalgia, chronic pancreatitis, severe angina pectoris, or other chronic disorders.

\section{Requisites for optimal results}

In order to obtain good results with nerve blocks, the anaesthesiologist or other doctor using these procedures must assume the responsibility of a physician and not act only as a technician expert at inserting needles. Even when acting as a consultant skilled in nerve blocks, it is important that the anaesthesiologist has an insight into the pain problem (Bonica, 1953, 1958, 1959, 1974).

The second requisite is that the physician using this method must have ample knowledge of various pain syndromes including the mechanisms and nociceptive pathways involved, the pathophysiology and symptomatology. It is essential to know the advantages, disadvantages, limitations and complications of the many therapeutic modalities that may be applicable for each syndrome. Only with this broad perspective can the best treatment or combination of treatments be chosen for each patient with a specific pain problem.

Thirdly, the physician must be willing to devote the time and effort to examine the patient thoroughly and confirm the diagnosis. This is essential even if the patient is referred by a highly competent colleague who has already made the diagnosis. A detailed history and thorough physical examination not only provide additional information but affords an opportunity to become acquainted with the patient, to investigate his or her personality and, most important, to win the confidence of the patient. A careful neurologic examination provides not only useful information, but constitutes a baseline in evaluating the effects of the block. These basic principles apply to all patients, particularly those with complex pain problems.

Once a tentative diagnosis is made it must be decided if the blocks are to be used to gain information, to predict the effect of prolonged interruption, or for therapy. It is essential to avoid the haphazard use of this tool, because this may cause more harm than good.

Another important requisite is that the individual $c$ must be highly skilled in carrying out the appropriate $\overrightarrow{\bar{F}}$ procedure and have a thorough knowledge of the immediate and long-term effects of the agents used.c Patients with chronic pain are not good subjects in $\overline{\bar{c}}$ whom to practice nerve blocks. The skill should be acquired by first observing experts doing the blocks $\cong$ and performing them under their supervision. Inos patients with severe pain, especially complex chronic $\vec{\circ}$ pain, nerve blocks must be performed carefully with meticulous attention to anatomic detail, with utmost $\vec{\omega}$ gentleness and by using high quality equipment including sharp needles, syringes that are in good working order and well-fitting, and a block tray adequately stocked with other necessary instruments.

It is important to inform the patient what and how the procedure will be done, and what will be? accomplished by it. This information should bec provided during the initial visit and repeated just ${ }_{0}$ prior to the block. If the patient does not realize the procedure is only to gain information and may provide only temporary or no relief, he may beథ disappointed and may not return for further care. $\mathbb{\Phi}$ Moreover, the patient should be reassured that $\frac{3}{0}$ everything will be done to minimize discomfort, that $\frac{\Phi}{-}$ he will be warned before each step of the procedureg్g $\vec{\omega}$ carried out, and that he may ask for a brief rest at ary time he requests it. In addition, if repeated therape tic blocks are to be done, appropriate sedatives oro narcotics may be used prior and during the block.

If the procedure is to be done for diagnostic oro prognostic purposes depressant drugs should not be $\frac{\partial}{\varnothing}$ given because the patient must be alert to answer尺 questions. It is essential to localize exactly the $\overrightarrow{\vec{O}}$ involved nerve or nerves. This can be accomplished 3 by checking the position of the needle with an X-ray? or image intensifier with or without prior injection of a contrast medium. Moreover, in using diagnostic or믐 prognostic blocks three essential principles must be adhered to: (1) injecting small $(3-4 \mathrm{ml})$ amounts of solution to avoid diffusion to adjacent segments and preclude misleading information: (2) no decision should be made until three or more blocks produce 2 consistent responses; and (3) it is best to use local 음 anaesthetics of different duration and correlate the $D$ duration of the block with the duration of pain relief. Use of "placebo" block may be added to helpN్ determine the diagnosis.

During and following the block, it is essential to $\tilde{o}^{-}$ assess the results carefully. Observation of the pa- $\omega$ tient's reaction to the insertion of the small needles, the formation of intracutaneous wheals (for cutane-o ous analgesia), and other parts of the procedure help $\mathbb{\complement}$ in evaluating response to noxious stimuli. Following? the block, it is essential to ascertain that the nerve 
pathways have been interrupted by repeating the neurologic examination. When this is established, the effect of the block in terms of pain relief must be assessed and the inplications for pathogenesis considered. This may require a few hours, several days, and perhaps weeks of observation. The amount, type, and duration of relief should be carefully noted and recorded on the patient's chart. In addition to observation by the physician, the results should be evaluated by the patient, the family if available and, most importantly, by the nursing staff.

It is important that all concerned appreciate fully the fact that nerve blocks are not the panacea and have limitations in diagnosis and in predicting the effects of prolonged interruption. While they are effective in a significant percentage of properly selected patients, it is essential to use the results within the framework of all other information obtained. To make the final diagnosis solely on the results of one or even several blocks is hazardous and may subject the patient to a useless destructive operation.

Finally, it is essential the patient and physicians realize these procedures produce side effects that may cause potentially serious complications. Therefore, all prophylactic measures against undesirable side effects must be carried out. An absolute requisite is that, other than the superficial infiltration of 3-5 ml of local anaesthetic, no block should be done without an assistant present and resuscitative equipment ready for immediate use. This should include an intravenous infusion set up before commencing the procedure so that it is available for the prompt administration of drugs to combat systemic reactions, arterial hypotension, or high spinal anaesthesia.

\section{Role of nerve blocks in pain control}

\section{Local infiltration}

Infiltration or topical application of local anaesthetics are the simplest and most frequently used analgesic blocks in the treatment of pain. Simplicity and apparent innocuousness makes this a method of choice among physicians working in their office. By producing physicochemical interruption of nervous pathways almost at the very source of the nociceptive process, it effectively relieves the pain and other symptoms of many disorders (Table 1).

\section{Sympathetic blocks}

\section{Causalgia and other reflex sympathetic dystrophies}

These conditions have been described elsewhere (see Wynn Parry and Withrington, 1984, this issue) and will not be discussed again here.
TABLE 1. Indications for local anaesthetic infiltration

Myofascial pain syndromes (Bonica, 1953, 1957, 1958; Sola, 1981; Travell, 1976)

Severe muscle spasm (Alexander 1954; Bonica, 1953, 1957, 1959; Finneson, 1973)

Joint sprains (Bonica, 1953, 1957)

Acute bursitis (Bonica, 1953, 1959; Littler, 1980)

Tendonitis (Bonica, 1953, 1959; Littler, 1980)

Ligamentous strains*

Joint arthritis (Finneson, 1973)

Pain scars

Neuroma (Bonica, 1953; Churcher, 1978)

*Including sacroiliac and sacrococcygeal (coccydynia).

\section{Post-amputation pain syndromes}

Following amputation of an extremity, 80-90\% of patients report feeling a phantom limb (or part) immediately after surgery. Of these, $10-15 \%$ have persistent severe pain in the phantom limb or the stump or both (Bonica, 1953; Feinstein, Luce and Langton, 1954). The characteristics of phantom limb pain vary but two predominant types usually occur: (1) a burning and throbbing pain not unlike that of causalgia and the other reflex sympathetic dystrophies which the patient describes as if the hand or foot were held too close to a fire; and (2) extremely abnormal position of the phantom limb with the hand or foot held in a painful, twisted, cramped, rigid, or flexed posture from which the patient is unable to release it. Pain in the stump is of three predominant types: (1) a constant, diffuse, burning, throbbing pain similar to that of reflex sympathetic dystrophies; (2) paroxysm of lancinating, shooting discomfort with a segmental or peripheral nerve distribution; or (3) a combination of these. Stump pain is usually associated with vasomotor and sudomotor disturbance manifested by coldness, cyanosis, oedema, signs of vasoconstriction and excessive sweating.

In patients with predominantly burning, aching pain associated with vasomotor and sudomotor changes in the stump, sympathetic interruption with regional sympathetic block or Bier block are effective in relieving the symptoms temporarily and at times permanently (Bonica, 1953, 1959; Livingston, 1938; Melzack and Wall, 1980). Immediately after the block, the patient feels partial or complete relief of pain and a warming of the stump. Sometimes the patient also feels the cramped or twisted extremity relax and assume a normal position. If the block affords complete or good relief of pain, it should be repeated several times to confirm the results and ascertain the duration of pain relief. If relief is of progressively longer duration and significantly outlasts duration of the block, surgical sympathectomy should be seriously considered. 
Several comments apply to the use of sympathetic block for causalgia, reflex sympathetic dystrophy and post-amputation pain. First, the earlier the treatment, the better the prognosis for a cure of the pain. Second, if the patient experiences even partial relief, sympathetic interruption should be repeated because sometimes, when the first two or three blocks produce partial or no relief, subsequent blocks will relieve the pain. A series of blocks may be done at intervals of 2-4 days or weekly depending on the response. Third, when sympathectomy is indicated, it can be done either chemically or surgically depending on the patient's physical condition, severity of the disease, and the patient's attitude toward the techniques. Chemical sympathectomy with $7 \%$ phenol in Conray 420 or $50 \%$ alchohol produces sympathetic interruption for several weeks to several months and is especially useful in children or poor risk patients. In patients who are younger and in good physical condition, surgical sympathectomy is preferable. Finally, it is essential to ascertain that sympathetic interruption is complete. This is especially important in patients who derive complete relief of the burning pain with a local anaesthetic sympathetic block or a Bier block but experience only partial or no relief following sympathectomy (Bonica, 1953, 1979). In such cases, it is likely that, while the local anaesthetic diffused widely to involve the sympathetic chain and anomalous sympathetic pathways (that often are present in the lower cervical and upper thoracic chain and the lumbar region), the operation was not extensive enough and/or it did not include the anomalous pathways.

Peripheral vascular disease. For nearly half a century, sympathetic interruption achieved either by regional sympathetic block or by chemical or surgical sympathectomy was considered one of the most important methods of managing patients with certain peripheral vascular diseases such as Raynaud's disease and chronic occlusive arterial disease (Buerger's disease). This is due to the fact that in many of these conditions, there is increased sympathetically-induced vasoconstriction with the consequent sequence of ischaemia, tissue damage, pain and trophic changes that can be partly or wholly reversed by early sympathetic interruption. However, the advent of effective surgical therapy using bypass grafts and other procedures and the widespread use of anticoagulant therapy have decreased the role of sympathetic blocks for this group of disorders.

Visceral pain. Block of the sympathetic nerves to thoracic or abdominal viscera is used to relieve severe visceral pain not amenable to other therapies or as an important adjunct to these. The basis for this is well known. The nociceptive pathways from the viscera accompany the efferent sympathetic nerves. Block with a local anaesthetic or neurolytic $\stackrel{\otimes}{\circledR}$ agent not only relieves pain but also interrupts the $C$ afferent and efferent limb and abnormal viscerovisceral and viscerosomatic reflexes that often de- $\stackrel{?}{\rightarrow}$ velop and contribute to the pathogenesis (Bonica, 1953, 1959, 1981). Segmental reflexes produce skel- 음 etal muscle spasm and sympathetic hyperactivity, $\frac{\pi}{\sigma}$ and this is further aggravated by suprasegmental $\propto$ reflexes that stimulate hypothalamic autonomic $\%$ centres and increase general sympathetic tone and $\vec{O}$ catecholamine release. All of these responses increase cardiac output and blood pressure, the work- $\vec{\omega}$ load of the heart, metabolism and oxygen consumption. Unless the severe pain and associated reflex responses are promptly eliminated, they be- 3 . come abnormal and greatly aggravate the patho- 8 physiology. Although potent narcotics administered in appropriate doses and by the appropriate route $\vec{\circ}$ produce adequate pain relief, they do not eliminate abnormal reflex responses. In contrast, block of the nociceptive pathways using local anaesthetics $\frac{\circ}{\square}$ blocks the afferent limb and thus obviates the reflex responses. These comments are especially

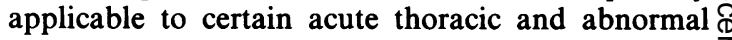
visceral painful conditions.

Acute myocardial infarction. As is well know acute myocardial infarction often produces severe excruciating pain and, unless promptly relieved, the aforementioned associated reflex responses may exäcerbate the myocardial pathophysiology (Bonica, 1953, 1981; Zanchetti and Malliani, 1974). The reflex responses may comprise either the Bezold-Jarish

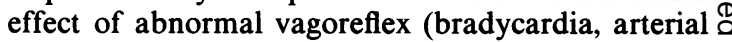
hypotension and atrioventricular block) or, more $\overrightarrow{\vec{A}}$ frequently, segmental and suprasegmental sympa- $\exists$ thetic hyperactivity with a consequent increase in cardiac output and myocardial oxygen consumption (Zanchetti and Malliani, 1974). Animal studies also suggest that segmentally induced sympathetic stimulation will produce reflex coronary vasoconstriction $\dot{0}$ that further impairs oxygen delivery to the myocardium (Feigl, 1975; Malliani, Schwartz and Zanchetti, of 1969; Zanchetti and Malliani, 1974). If this takes place in vessels perfusing myocardial tissue adjacent 음 to the infarcted muscle, it can result in making $D$ previously heathly myocardial tissue ischaemic and previously ischaemic tissue necrotic. Suprasegmental $\bar{N}$ reflexes stimulate autonomic centres and invariably further increase general sympathetic tone and cate- $N$ cholamine release (Strange et al., 1974; Zanchetti and $\underset{\omega}{N}$ Malliani, 1974). Moreover, the suprasegmental reflex responses are markedly enhanced by the severe anxiety that invariably develops in patients with acute myocardial infarction (Zanchetti and Malliani, $\stackrel{\oplus}{+}$ 1974). In addition, emotional stress may cause 0 
cortically mediated increased blood viscosity and clotting (Dreyfuss, 1956), fibrinolysis (Cash and Allen, 1967), and platelet aggregation (Zahvadi and Dreyfuss, 1967). The combined effects of segmental and suprasegmental reflexes, anxiety and stress greatly increase the workload of the heart and its oxygen consumption and, by segmental vasoconstriction and alteration of blood clotting, may further decrease the already compromised arteriosclerotic coronary circulation. This may markedly increase the discrepancy between oxygen supply and demand, and may cause extension of the infarction. It is therefore essential, promptly and effectively, to relieve the pain and anxiety, and so inhibit adverse reflex responses.

In patients with severe excruciating pain that does not respond to narcotics, cervicothoracic sympathetic block achieved with $12-15 \mathrm{ml}$ of $0.25 \%$ bupivacaine will produce effective analgesia for $8-10 \mathrm{hr}$ or more. Although the technique is often called 'stellate' ganglion block, this amount of local anaesthetic injected into the proper fascial plane will spread to involve the sympathetic chain from the middle cervical ganglian to the 4th or 5th thoracic ganglian. It thus blocks all sensory and sympathetic fibres to the heart. In patients with pain predominant on one side, a unilateral block suffices, but if the pain is bilateral, the block is done on the side with the most severe pain first and, after an interval of $30 \mathrm{~min}$, it is repeated on the opposite side. The value of sympathetic interruption in such cases is strongly suggested by controlled animal experiments. These demonstrate impressively that sympathetic denervation of the heart significantly reduces both size of experimentally induced infarction and animal mortality (Cox and Robertson, 1936; McEachern, Manning and Hall 1940; Schauer, Gross and Blum, 1937).

Angina pectoris. Angina pectoris when severe and intractable to medical therapy, was formerly managed with block of the upper four or five thoracic sympathetic ganglia with local anaesthetics and subsequently with alcohol (Bonica, 1953, 1957; Travell, 1976). However the advent of the coronaryaortic graft bypass operation has made chemical and surgical sympathectomy useless procedures. The only indications are patients with extensive coronary disease not amenable to the surgical procedure and in whom the anginal pain is disabling (Bonica and Benedetti, 1980). Local anaesthetic and neurolytic procedures may also be useful in relieving severe pain due to aortic aneurysm (Bonica, 1953; White, 1957).

Acute pancreatitis. This condition frequently causes severe or excruciating continuous pain, severe abdominal muscle spasm and rigidity, marked abdominal tenderness, nausea and vomiting, and moderate ileus with consequent abdominal distention. In most patients the pain and associated reflex responses impair pulmonary ventilation. Some patients develop progressive hypoxia and hypercapnia that may end in death. Although potent narcotics given intravenously partially relieve the pain, this condition is more effectively managed by regional block of the nociceptive afferents using splanchnic nerve block, coeliac plexus block or continuous segmental (T5T10) epidural block. Some writers (Gage, 1948) have suggested that in addition to relieving pain, interruption of nociceptive impulses decrease the severity and duration of the disease by combatting reflex spasm of the duodenum, sphincter of Oddi, and the entire ductal system (Feinstein et al., 1954). There is rapid release of extraductal pressure and toxic fluid is emptied from the extrabiliary and pancreatic ductal systems. The procedure also relieves the visceral vasospasm and reflex ileus.

Ureteral and biliary colic. These are among the most excruciatingly painful conditions experienced by some patients (Bonica, 1953, 1959). Although potent narcotics administered intravenously produce adequate pain relief, they increase spasm of the smooth muscle. On the other hand, block of nociceptive and efferent pathways with continuous segmental epidural block which involves T5-10 is highly effective in providing complete pain relief and relieving the associated reflex muscle spasm caused by biliary colic. Segmental block of T10-L2 is equally effective for ureteral colic. In some patients, the block also relaxes the ureter sufficiently to permit a stone to move down to a point where it can be removed through a cystoscope, thus obviating the need for an open operation (Bonica, 1953). An alternative method is paravertebral block of the splanchnic nerves and of the first and second lumbar ganglia.

Cancer pain. Neurolytic block of the splanchnic nerves or coeliac plexus achieved with $50 \%$ alcohol or $5-7 \%$ aqueous phenol is highly effective in relieving severe intractable pain caused by cancer of the pancreas, stomach, small intestine, gall bladder or other abdominal viscera, and is most effective in patients in whom the cancer has not spread to the parietal peritoneum. Moore et al. (1979) used this procedure in 168 patients and, of these, 157 (94\%) derived good to excellent pain relief and other benefits. These included less nausea and vomiting, increased food intake with occasional weight gain, improved bowel motility with the passing of flatus and stool, and elimination or marked reduction in the doses of narcotics needed. Block of the splanchnic nerves or coeliac plexus first with local anaesthetic and subsequently with alcohol or phenol may also be indicated in patients with severe intractable pain of 
chronic pancreatitis, postcholecystectomy syndrome or other chronic abdominal visceral diseases unrelieved by medical or surgical therapy (Bonica, 1953, 1959, 1981).

\section{Other indications for sympathetic blocks}

Herpes zoster and postherpetic neuralgia. These conditions have been effectively treated with block of the appropriate sympathetic pathways (Colding, 1969; Gale, 1973; Dan et al., 1979). Although controlled studies have not been done, there is some evidence that for older patients and those in whom sympathetic block has relieved symptoms in the acute stage, there is a reduction in the incidence of postherpetic neuralgia. In view of the fact that postherpetic neuralgia is one of the most difficult problems to treat, these procedures should be used early in the course of acute herpes zoster. In established postherpetic neuralgia and hyperpathia, results with sympathetic blocks are equivocal, but in view of the difficulties with this condition, blocks may be tried (Bonica, 1953, 1981).

Cancer pain. Sympathetic blocks may also be effective in relieving the burning, aching discomfort experienced by some patients with cancer of the face and head. Moreover, sympathetic blocks of the upper or lower limb are indicated in patients in whom cancer infiltration or compression of the brachial or lumbosacral plexus produces the symptomatology characteristic of reflex sympathetic dystrophy (Bonica, 1981; Hupert, 1979).

\section{Block of cranial nerves}

Block of the cranial nerves is useful in managing severe pain in the anterior two-thirds of the head. Local anaesthetic blocks are used for diagnosis or to predict effects of prolonged interruption achieved with neurolytic agents or neurosurgical ablative procedures. Alcohol block of one of the branches of the trigeminal nerve or Gasserian ganglion has long been used in patients with tic douloureux or severe cancer pain who are not suitable for neurosurgical operations (Bonica, 1953, 1959; Bonica and Madris, 1979). Although the advent of carbamazine, the recent reintroduction of thermocoagulation of the Gasserian ganglion and percutaneous differential radiofrequency rhizotomy of the trigeminal sensory root have all decreased the use of alcohol block, there is still a definite place for this in managing cancer pain and, to a lesser extent, in managing trigeminal neuralgia. Properly done, alcohol block of the Gasserian ganglion or sensory root produces pain relief in over $85 \%$ of patients with cancer pain in the anterior two-thirds of the head (Bonica, 1953, 1959;
Bonica and Madris, 1979). Block of the glossopharyngeal nerve alone or in combination with the vagus $\varnothing$ nerve below the jugular foramen is a useful diagnos-.. tic and prognostic procedure in patients with glosso- $\overrightarrow{\bar{s}}$ pharyngeal neuralgia or cancer pain of the throat prior to ablative section or percutaneous differential radiofrequency rhizotomy (Bonica, 1953; 1959; Bonica and Madrid, 1979).

\section{Paravertebral somatic nerve block}

Paravertebral block of one or more of the spinal nerves is a useful procedure in managing painful disorders of the back of the head, neck, trunk, and lower limbs (Bonica, 1953, 1959, 1984). Since this procedure includes the recurrent nerve and posterior division and the branches that supply the vertebra, the facet joint and the meninges, it is useful to help determine nociceptive pathways in patients with segmental neuralgia due to vertebral pathology such as osteoporosis, scoliosis or herniated intervertebral disc (Bonica, 1953, 1959, 1984).

Paravertebral somatic nerve block with local anaesthetics usually produces only temporary pain relief and is therefore most useful in acute conditions. Alcohol injection to produce prolonged interruption is absolutely contraindicated except in cancer patients with a short life expectancy. There are two reasons for this: (1) alcohol injection produces a postinjection. chemical neuropathy that eventually results in severo neuralgia that may be more uncomfortable than the original pain; (2) the weakness of paralysis caused by prolonged motor block may interfere with functional movement of the limbs.

\section{Intercostal block}

Intercostal nerve block is one of the most useful procedures for relief of a severe acute posttraumatic, postoperative or postinfectious pain in the thoracic or abdominal wall, and is highly effective in relieving severe pain from fracture of one or more ribs or of the sternum, dislocation of the costochondral junction, slipped rib cartilage, contusion chest pain, pleurisy and acute herpes zoster. It is a useful diagnostic/therapeutic procedure in extrapment of the intercostal 9 nerves in the rectal sheath said to be a frequent cause $D$ of abdominal pain and occasional chest pain (Applegate, 1972). Perhaps the most frequent use of $N$ intercostal block is to relieve severe pain following cholecystectomy, gastrectomy, mastectomy, thoraco- O tomy and sternotomy (Bonica and Benedetti, 1980; $\mathrm{\omega}$ Moore, 1975; Nunn and Slavin, 1980). A number of studies have shown the superiority of intercostal 0 nerve block over narcotics in managing postoperative pain (Bonica, 1984).

Intercostal block produces analgesia 2-4 times the 
duration of that achieved with the same drug dose injected into the epidural space. Moore et al. (1978) reported that following intercostal block with $4 \mathrm{ml}$ of $0.25 \%$ bupivacaine with adrenaline, analgesia lasted 10-12 hr. This makes it practical to induce intercostal block in the morning and have the patient ambulate, cough and be as active as possible during the analgesia that usually persists for the remainder of the day. If necessary, the block can be repeated in the evening, or at least each morning. Although intercostal block carries a risk of pneumothorax, skilfully done the incidence of this complication is less than $1 \%$ (Bonica, 1984).

\section{Peripheral nerve block}

Block of the brachial plexus or one or more of its major branches, and block of the lumbosacral plexus or the sciatic, femoral and obturator nerves may be used as a diagnostic or prognostic measure. Since all sympathetic fibres destined for the hand, forearm and low two-thirds of the arm are carried by the nerves derived from the brachial plexus, block of this structure is an effective measure to confirm the results of cervicothoracic sympathetic block in patients with reflex sympathetic dystrophy or those with painful peripheral vascular disorders. It is also useful in providing temporary relief of severe acute pain following trauma or operation, or in patients with severe vasospasm caused by accidental intra-arterial injection of such agents as thiopental and those with severe pain consequent to an embolus. Continuous brachial plexus block is especially useful in patients who have undergone reattachment of a severed limb or digits, and those where blood supply to the extremities is compromised (Rosenblatt, PepitoneRockwell and McKillop, 1979). In such circumstances, prolonged sympathetic block and analgesia enhance survival of the limb and concomitantly provide pain relief.

The indications for block of the sciatic and femoral nerves are similar to those of the brachial plexus. These may be used to temporarily control acute pain and produce complete sympathetic interruption of the foot and leg. Block of the lateral femoral cutaneous nerve is used to manage patients with meralgia paresthetica (Bonica, 1953; 1959). Obturator nerve block may be used in the management of abductor muscle spasm and differential diagnosis of patients with a painful hip (Bonica, 1953, 1959). A significant drawback to blocks of the somatic nerves to the extremities is the weakness and/or paralysis and loss of proprioception, touch and sensation that produce a useless limb. Therefore, except in extreme cases of patients with terminal cancer pain, prolonged blocks with alcohol or other neurolytic agents are absolutely contraindicated.

\section{Segmental epidural block}

Continuous segmental epidural block is one of the most practical techniques of managing patients with acute and chronic pain because placing a catheter at different levels of the extradural space can produce segmental analgesia involving two, three or as many as 10 spinal segments, in virtually any part of the body, and usually involves somatic and visceral nerves. It is one of the most effective methods to relieve severe pain of acute pancreatitis, biliary colic, renal and ureteral colic, multiple rib fractures, and other severe post-traumatic pain, and in controlling postoperative pain in the thorax, abdomen or lower limbs. It is used to provide temporary relief of severe pain due to herniated intervertebral discs or caused by vertebral or pelvic fracture because it not only provides complete relief (in contrast to the partial relief achieved with narcotics) but also relieves the reflex muscle spasm and permits more definitive treatment.

\section{Subarachnoid block}

Subarachnoid neurolysis achieved by injection of small amounts of alcohol or phenol into the subarachnoid space is one of the most effective methods for the relief of severe intractable pain below the neck (Bonica, 1953, 1959; Ferrer-Brechner, 1981; Hay, 1962; Swerdlow, 1979; Wood, 1978). Pain relief lasts for several days to several months, and sometimes longer, although frequently it is necessary to do several blocks to effect prolonged relief. Numerous reports suggest that neurolytic subarachnoid block produces complete relief in $50-60 \%$ of cancer patients, partial relief in $20-25 \%$, and no relief in the rest (Bonica, 1953, 1959; Hay, 1962; Swerdlow, 1979). This compares favorably with the results achieved with neurosurgical procedures. With subarachnoid block of the roots supplying the upper limb, there is a $15-20 \%$ incidence of muscle weakness. If the block is done to relieve pain in the pelvis or lower limbs, there is a $20-25 \%$ incidence of bladder and/or rectal dysfunction and lower limb muscle weakness.

\section{References}

AleXANDer, F.A.D. (1954) Control of pain. In: Anesthesiology. (Ed. D. Hale), Ch. 28. Davis, Philadelphia.

APPLEGATE, W.V. (1972) Abdominal cutaneous nerve entrapment syndrome. Surgery, 71, 118.

BoniCA, J.J. (1953) Management of Pain. Lea \& Febiger, Philadelphia.

BONICA, J.J. (1957) Management of myofascial pain syndromes in general practice. Journal of the American Medical Association, 165, 732.

BoNICA, J.J. (1958) Diagnostic and therapeutic blocks. A reappraisal based on 15 year's experience. Anesthesiology and Analgesia, 37, 58.

BoNiCA, J.J. (1959) Clinical Applications of Diagnostic and Therapeutic Nerve Blocks. Charles C. Thomas, Springfield. 
BonICA, J.J. (1974) Current role of nerve blocks in the diagnosis and therapy of pain. In: Advances in Neurology, Vol. 4. (Ed. J. J. Bonica), p. 445. Raven Press, New York.

BoNICA, J.J. (1979) Causalgia and other reflex sympathetic dystrophies. In: Advances in Pain Research and Therapy, Vol. 3. (Eds. J. J. Bonica, J. C. Liebeskind and D. Albe-Fessard). Raven Press, New York.

BoniCA, J.J. (1981) Blocks of the Sympathetic Nervous System, Vol. 1. (1980), Vol. 2. (1981). Frank J. Corbett, Inc., Chicago.

BoNICA, J.J. (1984) Local anaesthesia and regional blocks. In: Textbook of Pain. (Eds. P. D. Wall and R. Melzack). Churchill Livingstone, London.

BonicA, J.J. \& BenedetTl, C. (1980) Postoperative Pain. In: Surgical Care: A Physiological Approach to Clinical Management. (Eds. R. E. Concon and J. J. deCosse), p. 394. Lea \& Febiger, Philadelphia.

BoNICA, J.J. \& MADRIS, J.L. (1979) Cancer pain in the head and neck. Role of nerve blocks. In: Advances in Pain Research and Therapy. Vol. 3. (Eds. J. J. Bonica, J. C. Liebeskind and D. AleFessard,). Raven Press, New York.

CASH, J.D. \& ALLAN, A.G.E. (1967) Effect of mental stress on the fibrinolytic reactivity to exercise. British Medical Journal, 2 545

Churcher, M. (1978) Peripheral nerve blocks in the relief of intractable pain. In: Relief of Intractable Pain. (Ed. M. Swerdlow). Excerpta Medica, Amsterdam.

Colding, A. (1969) The effect of regional sympathetic blocks in the treatment of herpes zoster. Acta Anesthesiologica Scandinavica, 13, 133.

Cox, W.V. \& Robertson, H.F. (1936) The effect of stellate ganglionectomy on the cardiac function of intact dogs and its effect on the extent of myocardial infarction and on cardiac function following coronary artery occlusion. American Heart Journal, 12, 285.

Dan, K., TanakaK, H. \& Kamihara, Y. (1979) Herpetic pain and T-cell subpopulation. In: Advances in Pain Research and Therapy, Vol. 3. (Eds. J. J. Bonica, J. C. Liebeskind and D. Albe-Fessard) Raven Press, New York.

DREYFUSS, F. (1956) Coagulation time of the blood, level of blood eosinophils and thrombocytes under emotional stress. Journal of Psychosomatic Research, 1, 252.

DRUCKer, W.R., Hubay, C.A., Holden, W.D. \& Bukovnik, J.A. (1959) Pathogenesis of post-traumatic sympathetic dystrophy. American Journal of Surgery, 97, 353

FEIGL, E.O. (1975) Control of myocardial oxygen tension by sympathetic coronary vasoconstriction in the dog. Circulation Research, 37, 88.

FEINSTEIN, D., LUCE, J.C. \& LANGTON, J.N.K. (1954) The influence of phantom limbs. In: Human Limbs and Their Substitutes. (Eds. P. E. Klopsteg and P. D. Wilson), p. 79. McGraw Hill, New York.

FERRER-BRECHNER, T. (1981) Epidural and intrathecal phenol neurolysis for cancer pain. Anestheology Review, 8, 14.

Finneson, B.E. (1973) Low Back Pain. J. B. Lippincott, Philadelphia.

GAGE, I.M. (1948) Treatment of acute pancreatitis. Surgery, 23, 723.

GALE, D.A. (1973) The management of neuralgias complicating herpes zoster. The Practitioner, 210, 794.

HAY, R.C. (1962) Subarachnoid alcohol block in the control of intractable pain. Anesthesia and Analgesia Current Research, 41, 12.
HUPERT, C. (1979) Recognition and treatment of causalgic pain occuring in cancer patients. In: Advances in Pain Research and 0 Therapy, Vol. 3. (Eds. J. J. Bonica, J. C. Liebeskind and D. Albe- $؟$ Fessard,). Raven Press, New York.

LITTLER, T.R. (1980) Pain relief in rheumatic condition. Part I. In: $\overrightarrow{\overline{\vec{C}}}$ Persistent Pain: Modern Methods of Treatment, Vol. 2, (Ed. S. Lipton). Academic Press, London.

Livingston, W.K. (1938) Post-traumatic pain syndrome. Western Journal of Surgery, Obstetrics and Gynecology, 46, 341 .

Malliani, A., SchwartZ, P.J. \& Zanchetti, A. (1969) A sympathetic reflex elicited by experimental coronary occlusion. American Journal of Physiology, 217, 703.

MCEAChern, C.G., ManNing, G.W. \& Hall, G.E. (1940) Sudden occlusion of coronary arteries following removal of cardiosensory pathways. Internal Medicine, 65, 661.

MElzaCK, R.W. \& WALl, P.D. (1980) Challenge of Pain. Basic Books, New York.

MOORE, D.C. (1975) Intercostal nerve block for postoperative somatic pain following surgery of thorax and upper abdomen. British Journal of Anaesthesia, 47, 284.

MOORE, D.C. (1979) Celiac (splanchnic) plexus block with alcohol for cancer pain of the upper intra-abdominal viscera. In: Advances in Pain Research and Therapy, Vol. 2. (Eds. J. J. Bonica and V. Ventafridda). Raven Press, New York.

MoORe, D.C., Bridenbaugh, L.D., Thompson, C.E., Balfour, R.I. \& HORTON, W.C. (1978) Bupivacaine; A review of 11,0800 cases. Anesthesia and Analgesia, 57, 42.

NUNN, J.F. \& SLAVIN, G. (1980) Posterior intercostal nerve block for pain relief after cholecystectomy: Anatomic basis and efficacy. British Journal of Anaesthesia, 52, 253.

Rosenblatt, R.M., Pepitone-Rock Well, F. \& McKillop, C. (1979) Continuous axillary analgesia for traumatic hand surgery Anesthesiology, 51, 565.

Schauer, G., Gross, L. \& Blum, L. (1937) Hemodynamic studie: in experimental coronary occlusion. IV. Stellate ganglionecto experiments. American Heart Journal, 14, 669.

SolA, A.E. (1981) Myofascial trigger point therapy. Resident and Staff Physician, 27, 38.

Strange, R.C., Vetter, N., Rowe, M.J. \& Oliver, M.F. (1974) Plasma cyclic AMP and total catacholamines during acute myocardial infarction in man. European Journal of Clinical Investigation, 4, 115.

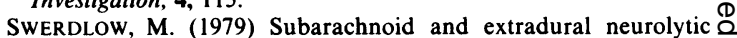
blocks. In: Advances in Pain Research and Therapy, Vol. 2. (Ed. $\overrightarrow{\bar{O}}$ J. J. Bonica and V. Ventafridda), p. 325. Raven Press, New York.

TRAVell, J. (1976) Myofascial trigger points. In: Advances in Pain Research and Therapy, Vol. 1. (Eds. J. J. Bonica and D. AlbeFessard). Raven Press, New York.

WHITE, J.C. (1957) Cardiac pain: Autonomic pathways and phy:iological mechanisms. Circulation, 16, 644.

WooD, K.M. (1978) The use of phenol as a neurolytic agent: $A$ review. Pain, 5, 105

Wynn Parry, C.B. \& Withrington, R.H. (1984) Painful disorders of peripheral nerves. Postgraduate Medical Journal, 60, 869

ZahVADI, A. \& DREYfuss, F. (1967) Second Congress of the International Society of Thrombosis and Hemostasis, Oslo, Norway.

ZanChetTI, A. \& Malliani, A. (1974) Neural and psychological factors in coronary disease. Acta Cardiologica, 20, (Suppl.), 69. 